\title{
Utility of multispectral imaging for nuclear classification of routine clinical histopathology imagery
} Laura E Boucheron ${ }^{* 1,2}$, Zhiqiang Bi ${ }^{1}$, Neal R Harvey ${ }^{2}$, BS Manjunath ${ }^{1}$ and David L Rimm ${ }^{3}$

Address: ${ }^{1}$ Electrical and Computer Engineering Department, University of California, Santa Barbara, CA, 93106, USA, ${ }^{2}$ Space and Remote Sensing Sciences, Los Alamos National Laboratory, P.O. Box 1663, Los Alamos, NM, 87545, USA and ${ }^{3}$ Department of Pathology, Yale University School of Medicine, P.O. Box 208023, New Haven, CT, 06520, USA

Email: Laura E Boucheron* - lauraeb@ece.ucsb.edu; Zhiqiang Bi - zb26@ece.ucsb.edu; Neal R Harvey - harve@lanl.gov; BS Manjunath - manj@ece.ucsb.edu; David L Rimm - david.rimm@yale.edu

* Corresponding author

from 2006 International Workshop on Multiscale Biological Imaging, Data Mining and Informatics

Santa Barbara, CA, USA. 7-8 September 2006

Published: 10 July 2007

BMC Cell Biology 2007, 8(Suppl I):S8 doi:10.1 I86/147I-2I2I-8-SI-S8

This article is available from: http://www.biomedcentral.com/I47I-2I2I/8/SI/S8

(c) 2007 Boucheron et al; licensee BioMed Central Ltd.

This is an open access article distributed under the terms of the Creative Commons Attribution License (http://creativecommons.org/licenses/by/2.0), which permits unrestricted use, distribution, and reproduction in any medium, provided the original work is properly cited.

\begin{abstract}
Background: We present an analysis of the utility of multispectral versus standard RGB imagery for routine H\&E stained histopathology images, in particular for pixel-level classification of nuclei. Our multispectral imagery has 29 spectral bands, spaced $10 \mathrm{~nm}$ within the visual range of 420-700 $\mathrm{nm}$. It has been hypothesized that the additional spectral bands contain further information useful for classification as compared to the 3 standard bands of RGB imagery. We present analyses of our data designed to test this hypothesis.

Results: For classification using all available image bands, we find the best performance (equal tradeoff between detection rate and false alarm rate) is obtained from either the multispectral or our "ccd" RGB imagery, with an overall increase in performance of $0.79 \%$ compared to the next best performing image type. For classification using single image bands, the single best multispectral band (in the red portion of the spectrum) gave a performance increase of $0.57 \%$, compared to performance of the single best RGB band (red). Additionally, red bands had the highest coefficients/ preference in our classifiers. Principal components analysis of the multispectral imagery indicates only two significant image bands, which is not surprising given the presence of two stains.
\end{abstract}

Conclusion: Our results indicate that multispectral imagery for routine H\&E stained histopathology provides minimal additional spectral information for a pixel-level nuclear classification task than would standard RGB imagery.

\section{Background}

The use of multispectral imaging capabilities is relatively new to the field of cyto- and histo-pathology, particularly for transmitted brightfield microscopy $[1,2]$. Recent publications (e.g., [3-6]) have begun to explore the use of extra information contained in such spectral data (29-33 
wavelengths in the visible spectrum, from $400 \mathrm{~nm}$ to 720 $\mathrm{nm}$, spaced $10 \mathrm{~nm}$ apart), in particular for multiply stained ( $>2$ stains) specimens. Specifically, there have been comparisons of spectral unmixing algorithms (to separate constituent dyes) which demonstrate the advantage of multispectral data [5,7]. The added benefit of multispectral imaging for analysis of routine H\&E cyto/ histopathology imagery, however, is still largely unknown, although some promising results are presented in [6].

While the use of multispectral light microscopy is new to cyto/histopathology, many researchers have used single or dual narrow-band filters to enhance imagery for particular stains, most using a red filter (or the red channel of an RGB image) for enhancement of Hematoxylin or Feulgen staining [8-12], and some using a green filter for enhancement of Feulgen staining [13-16].

We present analyses of our multispectral data designed to test the hypothesis that the additional spectral bands contain more information useful for classification as compared to the 3 standard bands of RGB microscopy imagery. The work presented here is an extension of the work presented in [17].

\section{Results and discussion Classification using all image bands}

We split our dataset in (approximately) half to create a set of training images and a set of test images; half each of the benign and malignant subsets were randomly assigned to the training or test set to allow for even representation of benign and malignant characteristics in both sets. (One less benign image is included in the training set.) Applying all six classifiers to each image, using all available image bands, and averaging over the images contained in the test (out-of-sample) set, we achieve the results shown in Figure 1. Since the AFE tool GENIE is stochastic, we average ten independent runs. We would like to point out that the quadratic SVM (NLSVM) was run with only $10 \%$ of the total training data.

The best performance $P$ is obtained with either the multispectral or ccd image stacks, with ML and AFE performing better with ccd imagery, and MED, FLDA, SAM, and both SVMs performing better with multispectral. It is important to note, however, that these increases in performance are only, on average, $0.79 \%$. We use a paired Wilcoxon signed rank test to determine the statistical significance of these differences in performance, and show our results in Table 1 ; we see that less than half of these differences are statistically significant.

We have shown in this section, using a pairwise Wilcoxon signed rank test, that only a few performance differences between multispectral and RGB imagery are statistically significant. Furthermore, we note that these statistically significant differences are $0.46 \%, 0.76 \%$, and $0.38 \%$ increase in favor of multispectral imagery over rgbequal, truecolor, and ccd, respectively, for MED; $0.32 \%$ in favor of multispectral over rgbequal for SAM; $0.58 \%$ in favor of multispectral over rgbequal and $0.35 \%$ in favor of ccd over multispectral for AFE; $1.06 \%$ in favor of multispectral over rgbequal for LSVM; and $1.7 \%, 1.1 \%$, and $1.1 \%$ in favor of multispectral over rgbequal, truecolor, and ccd, respectively, for NLSVM.

\section{Classification using single image bands}

To gain a better understanding of the relative contributions of specific image bands, we apply the ML, MED, FLDA, and AFE classifiers to each individual image band for each image type. We exclude the SAM classifier here since it will fail on one-band images, and we exclude the SVM for computational reasons (it would be prohibitively computationally intensive to optimize kernel parameters for each image band). Performance scores for classification using single multispectral bands are shown in Figure 2A. Here we see the best performance scores occurring in the red portion of the spectrum, with poorer performance in the lower green portion and at the extremes of the spectrum.

Similarly, we note that for RGB images, the red channels yield the best performance (Figure 2B); we choose the AFE classifier for presentation here since it consistently yields the highest performance scores, though the other three classifiers display the same trends. While it may seem contradictory that in RGB imagery the green channel outperforms the blue channel when the opposite is true in multispectral imagery, it is important to remember how the multispectral bands are allocated to each of the RGB bands. Consider, for example, the allocation of bands in rgbequal imagery: the bands from $510 \mathrm{~nm}$ to $600 \mathrm{~nm}$ are averaged to yield the green channel. Referring to Figure 2A we see that these bands have a large variation in performance. Thus, to obtain the green channel, we are averaging multispectral bands, several of which have relatively good performance. A similar situation occurs with the truecolor and ccd imagery, albeit with a weighting applied to each band.

We find the analysis of performance on single image bands satisfactory from an intuitive standpoint. Since the nuclei are stained with the blue-colored Hematoxylin which will block red light, the red portions of the spectrum have the best contrast and perform the best for this nuclear classification task. While green light is also blocked by the Hematoxylin, so also is it blocked by the Eosin, rendering the green portion of the spectrum less informative for the task at hand. 


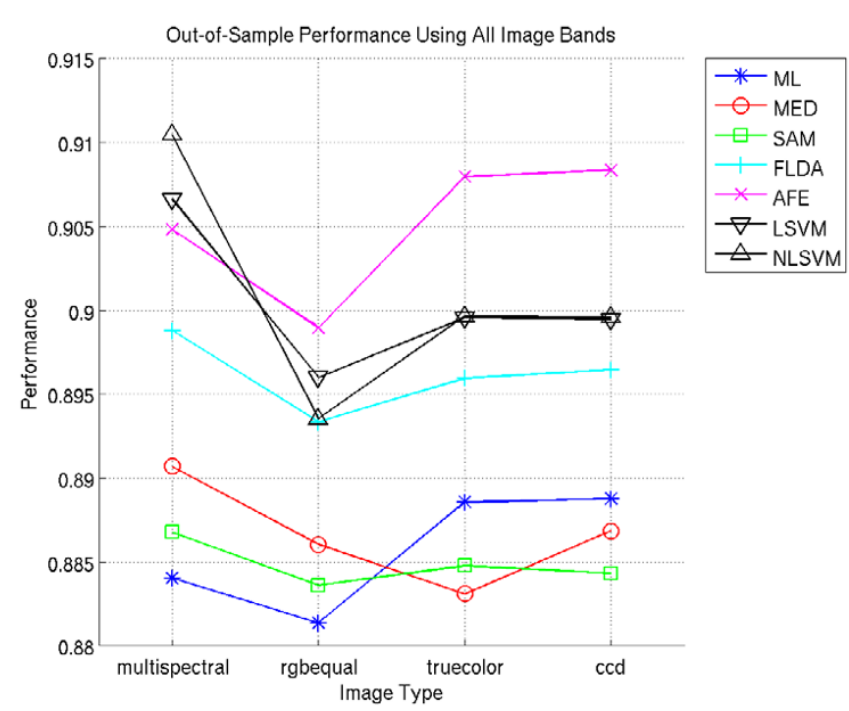

Figure I

Average performance using all available image bands. Performance (Equation I) is presented within the range $[0, \mathrm{I}]$.

The distinction in performance of red channels between the RGB image types is not large; we do note, however, that the single best performing multispectral band yields a performance increase of $0.57 \%$ as compared to the single best RGB band, averaged over all 4 classifiers. This performance increase is consistently in favor of single multispectral image bands, but are not generally statistically significant (refer to Table 2).

We have shown in this section that performance differences between single multispectral image bands and single RGB image bands are not statistically significant. This would seem to indicate that the individual multispectral image bands are not yielding any more specific spectral information than are the individual RGB image bands for this nuclear classification task.

\section{Analysis of FLDA coefficients and bands chosen in AFE solutions}

We expect that the single image bands which yield the best performance should also be the bands used most often by the classifiers. A direct examination of this is possible with the FLDA and AFE classifiers. For FLDA, image bands are weighted and summed; the higher the absolute value of the coefficient, the more important the image band. Plots of these coefficients for multispectral and RGB imagery are shown in Figure 3. For the AFE classifier, more important image bands should be chosen more often in solutions; plots of the average number of times an image band is chosen in an AFE solution are shown in Figure 4, where the 10 independent runs have been averaged. Once again, in both the FLDA and AFE classifier, we note a preference for the red portion of the spectrum.

We note also that with RGB imagery, the FLDA classifier weights the red channel the most, followed by the blue, and finally green channels. Similarly, the AFE classifier chooses the red channel most often, followed in turn by blue and green. Comparing the multispectral plots for the AFE and FLDA classifiers, there are striking similarities in the relative use/weighting of bands, particularly in the red portion of the spectrum (i.e., $580-650 \mathrm{~nm}$ ). The more prevalent use of green and blue bands in the AFE classifier, compared to FLDA, may be due to the classifier's ability to extract local features, making those bands more useful beyond the raw spectral attributes used by the FLDA classifier. Overall, considering the disparate nature of these two classifiers, we find it very interesting that they both display similar preferences for particular image bands.

We use the analysis in this section as a complement to the analysis of performance on single image bands. Specifically, we have shown that image bands that yielded the better performances are also the image bands chosen preferentially in both the FLDA and AFE classifiers. While it may be more qualitatively satisfying if the plots of Figures 3 and 4 would bear more resemblance to those of Figure 2 , it is important to remember that these two analyses are

Table I: Wilcoxon p-values for performances of multispectral versus RGB imagery.

\begin{tabular}{lcccc}
\hline Classifier & Image & rgbequal & $\begin{array}{c}\text { Image } \\
\text { truecolor }\end{array}$ \\
\hline ML & multi & 0.5440 & 0.0978 & 0.0822 \\
MED & multi & $\mathbf{0 . 0 0 0 0}$ & $\mathbf{0 . 0 0 0 1}$ & 0.0000 \\
SAM & multi & $\mathbf{0 . 0 0 5 7}$ & 0.7343 & 0.8290 \\
FLDA & multi & 0.0656 & 0.0752 & 0.1156 \\
AFE & multi & $\mathbf{0 . 0 0 3 0}$ & 0.1109 & $\mathbf{0 . 0 2 8 5}$ \\
LSVM & multi & $\mathbf{0 . 0 0 1 2}$ & 0.6288 & 0.4284 \\
NLSVM & multi & $\mathbf{0 . 0 0 0 0}$ & $\mathbf{0 . 0 0 4 7}$ & $\mathbf{0 . 0 0 6 0}$
\end{tabular}

Wilcoxon paired signed-rank test p-values are presented to 5 significant digits, and bold entries correspond to statistical significance at the p-value of 0.05 . 

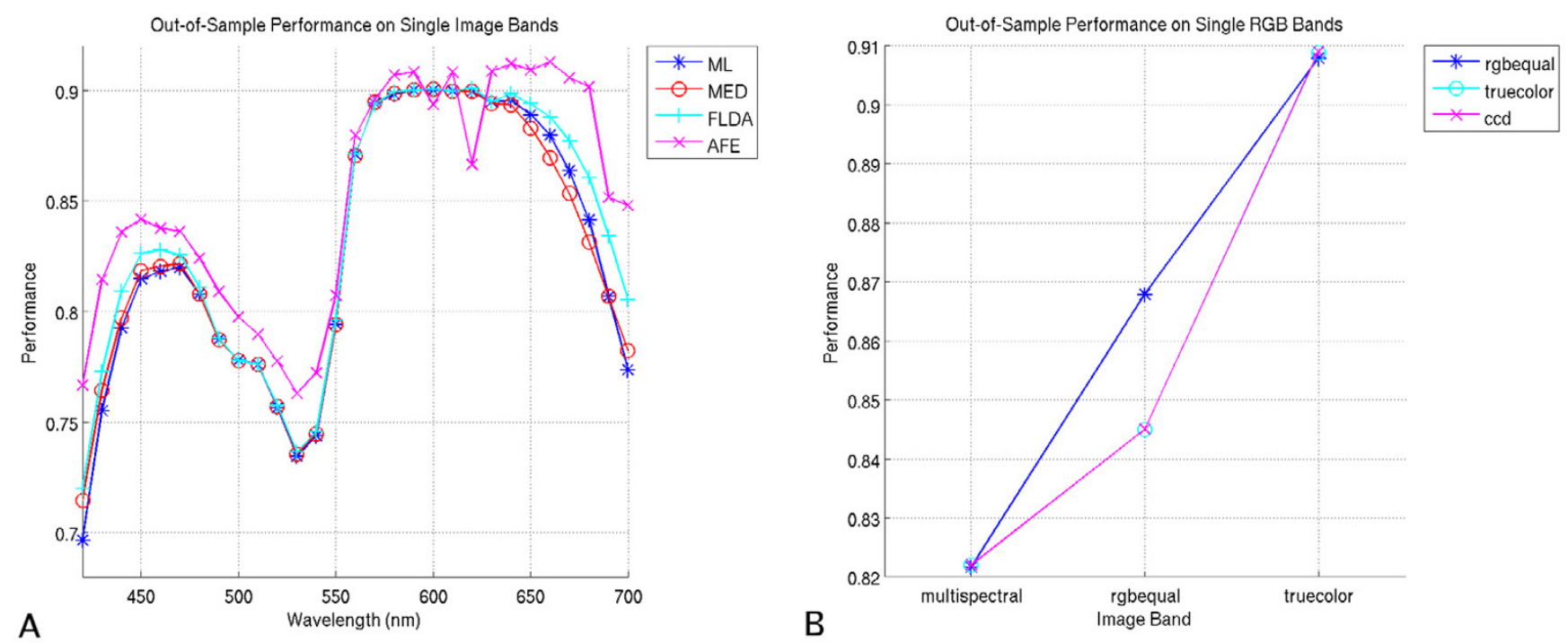

Figure 2

Performance on single image bands. (A) Out-of-sample performance scores on single multispectral bands. (B) Out-ofsample performance scores on single RGB bands for the AFE classifier.

very distinct from one another. In the case of Figure 2, we are limiting the classifiers to a single image band, and optimizing the performance, whereas for Figures 3 and 4 we are providing the classifiers with a choice of all available image bands and optimizing performance. As a more intuitive example, for the FLDA classifier, even if a specific image band $\mathrm{X}$ performs well when used alone, this same image band $\mathrm{X}$ may not yield as much information as, say, the linear combination of bands $\mathrm{Y}$ and $\mathrm{Z}$. We have shown, therefore, in this analysis, a classifier preference for image bands which yield better performance when used singly in classification.

\section{Principal components analysis of image stacks}

We use Principal Components Analysis (PCA) as a dimensionality reduction method to see how many "important" bands actually exist within our multispectral image stacks. We choose PCA rather than another dimensionality reduction technique, such as Independent Components Analysis (ICA), since PCA has a well established ranking for the resulting vectors. While there has been at least one ranking method suggested for ICA, the ratio of betweenclass to within-class variance [18], there is not a universally accepted ranking for ICA vectors. While ICA may yield a better separation of the independent causes in our data (i.e., the two stains), we are interested in the use of a dimensionality reduction technique mainly to help interpret the (lack of) differences in performance we have presented for our multispectral and RGB imagery.

As input to the PCA algorithm, we use the $(768 \cdot 896) \times 29$ matrix where the rows correspond to a single image pixel and the columns are the pixel values for each of the 29 multispectral image bands. We plot the average sorted eigenvalues of the covariance matrix of this input in Figure 5 , where for each image we normalize the eigenvalues so that the largest eigenvalue has unit value. We note that there appears to be one dominant eigenvalue, with the second ranked eigenvalue at approximately one-tenth the value of the dominant one; given the two stains in our his-

Table 2: Wilcoxon p-values for performances of the best multispectral band versus the red RGB channel.

\begin{tabular}{llccc}
\hline Classifier & Band & Band & rgbequal R \\
& & truecolor R & 0.3389 \\
ML & multi $590 \mathrm{~nm}$ & $\mathbf{0 . 0 3 1 6}$ & 0.3086 & 0.1714 \\
MED & multi $600 \mathrm{~nm}$ & $\mathbf{0 . 0 2 1 8}$ & 0.2452 & 0.3600 \\
FLDA & multi $620 \mathrm{~nm}$ & $\mathbf{0 . 0 0 1 7}$ & 0.2452 & 0.4653 \\
AFE & multi $660 \mathrm{~nm}$ & 0.0937 & 0.4048 &
\end{tabular}

Wilcoxon paired signed-rank test $\mathrm{p}$-values are presented to 5 significant digits, and bold entries correspond to statistical significance at the $\mathrm{p}$-value of 0.05 . 
topathology imagery, we expected two dominant eigenvalues. We show in Figure 6 the projection of an example image onto the first three eigenvectors. The first projection seems to highlight nuclear regions (i.e., the Hematoxylin), the second projection seems to highlight the connective tissue and cytoplasm (i.e., the Eosin), and the third and subsequent projections do not have any obvious correlation with the tissue stains.

We have thus found that PCA indicates the presence of 2 dominant eigenvalues, when we consider the principle components responsible for $97 \%$ of the variation in the data. This indicates the presence of only 2 informationbearing bands in the imagery for this nuclear classification task, providing insight into the approximately equivalent performance of the RGB imagery and multispectral. We have also shown that these 2 informative bands demonstrate a direct relationship to the two image stains. Interestingly, the first component is responsible for $93 \%$ of the total variation; this band is generally correlated with Hematoxylin, but is sometimes correlated instead with Eosin. The possibility that other image bands may contain important diagnostic information for further analysis is still an open question [2].

\section{Conclusion}

We have shown a demonstration of performance for different image types and different classifiers in a nuclear classification task. Results seem to indicate only slight performance differences (less than 1\%) using multispectral imagery as opposed to our derived RGB imagery; while these performance increases are small, we report them here since they are a direct result from our experiments, and may be statistically significant. These conclusions hold for both classification using all available image bands as well as using single image bands, indicating that the multispectral bands do not contain much more discriminatory spectral information than do the RGB bands for this nuclear classification task. There are, undoubtedly, a number of metrics that could be used in a study such as this, and we may have been able to find a metric for which multispectral would fare better (or worse) than presented here. However, we wanted to use a metric that provides an equal trade-off between two commonly used metrics (detection rate and false alarm rate). We have also shown that the single image bands with the best performance are the image bands chosen more often/weighted more heavily by the AFE and FLDA classifiers. Finally, we have shown through the use of PCA as a dimensionality reduction method, that only 2 image bands are carrying $97 \%$ of the variation in our image data, and appear to be correlated with the two image stains. This result provides some insight into the roughly equivalent performance of RGB imagery to multispectral. While the results presented here are intriguing, they are by no means complete, since we are considering only a single pixel-level classification task. Future work will continue to compare multispectral with RGB imagery for further classification tasks, as well as other image analysis tasks, including object-level analysis. In particular, we are currently researching methods to segment (i.e., delineate) individual nuclei using the results of these pixel-level classifications.
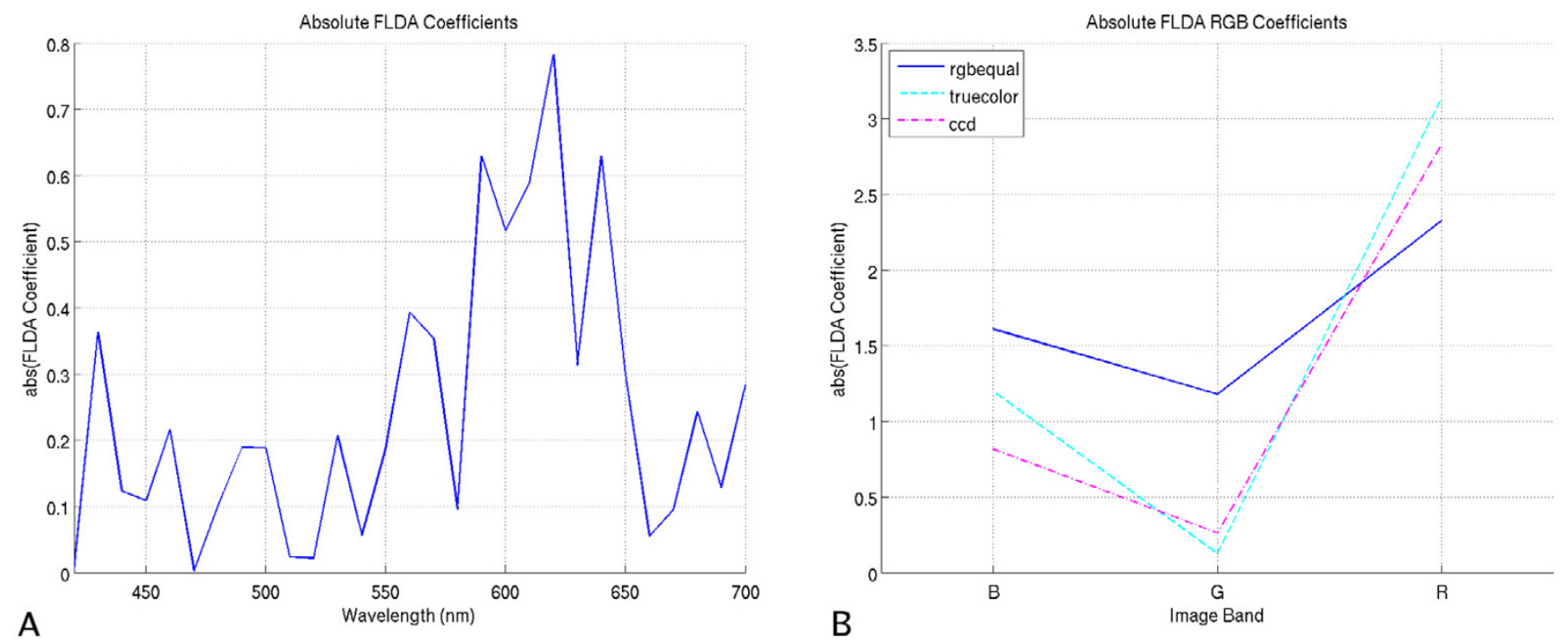

Figure 3

FLDA coefficients. (A) FLDA coefficients for multispectral imagery. (B) FLDA coefficients for RGB imagery. 

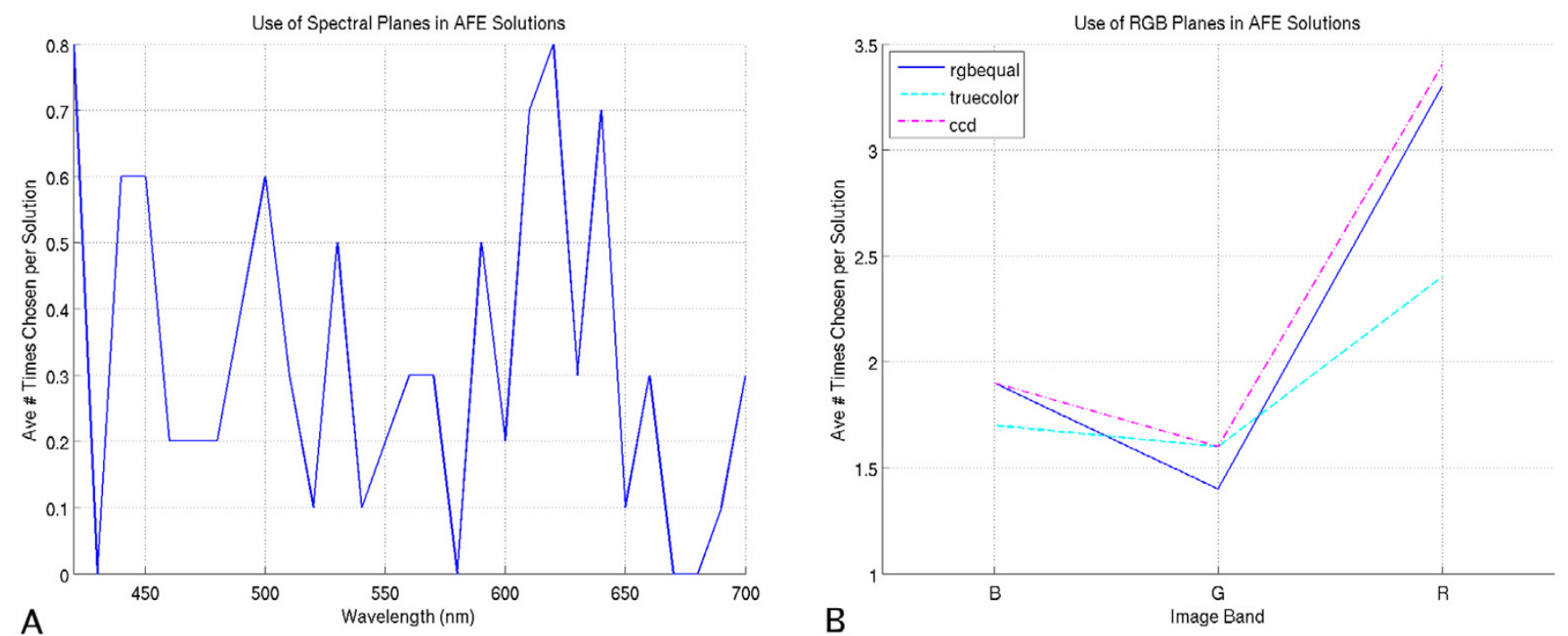

Figure 4

Spectral bands chosen in AFE solutions, averaged over 10 independent runs. (A) Multispectral bands chosen in AFE solutions. (B) RGB bands chosen in AFE solutions.

\section{Methods}

Sample preparation and image acquisition

Our dataset contains $58 \mathrm{H} \& \mathrm{E}$ stained histopathology images of breast tissue from the Yale Tissue Microarray Facility [19]. The data was captured from 5 microarrays (ytma10, 12, 49, and 55), with $(6,6,34$, and 6) images captured per array, respectively; in total we have 26 malig-

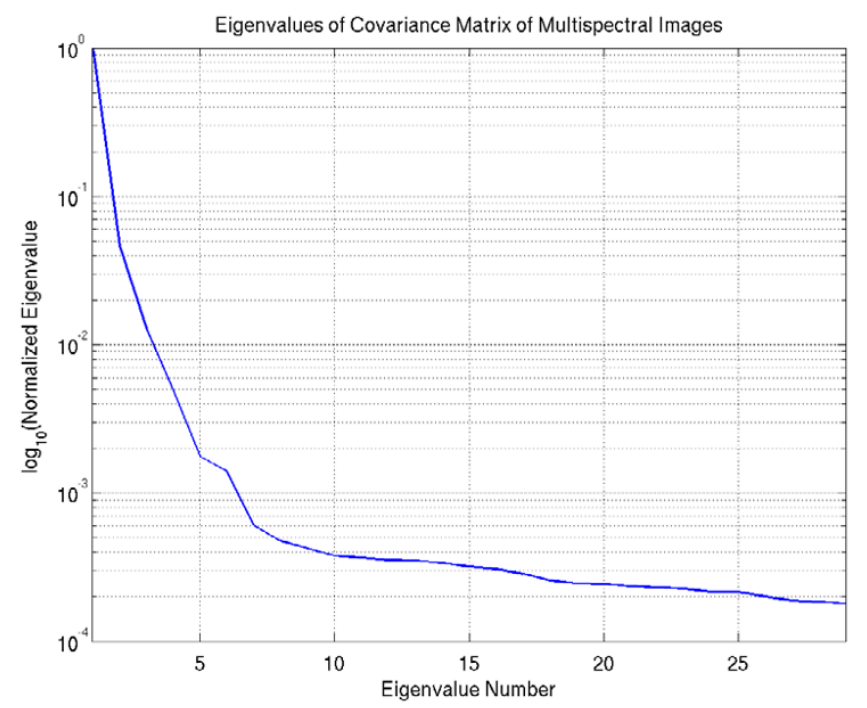

Figure 5

Logarithmic plot of the eigenvalues of multispectral imagery, from PCA. Eigenvalues for each image are normalized so that the largest eigenvalue has unit value. nant images, and 32 benign (including 6 normal from ytma55). Our 58 images are not microarray images in the general sense since we are dealing with single histopathology images as might be obtained from standard clinical biopsy specimens. The multispectral images have 29 bands, spaced $10 \mathrm{~nm}$ apart, ranging within the visible spectrum from 420 to $700 \mathrm{~nm}$, acquired using the VariS$\operatorname{pec}^{\mathrm{TM}}$ (CRi, Woburn, MA) liquid crystal tunable filter and a typical clinical pathology microscope setup with a $40 \times$ objective (400× total magnification). Each band is represented in an image stack as an 8 bit, $768 \times 896$ grayscale image; an example is shown in Figure 7. It should be noted that each image band has been corrected for illumination differences via a flat-fielding operation; this is part of the acquisition software included with the VariSpec ${ }^{\mathrm{TM}}$.

\section{Derivation of RGB imagery}

One could foresee many methods for the derivation of RGB imagery from multispectral. We use here:

1. rgbequal: created by (approximately) equally allocating the 29 bands to $R, G$, and B, similar to the approach in [7], reflecting a rough approximation of the three spectral ranges associated with the three colors red, green, and blue, albeit with some ambiguity in allocation of intermediate colors (e.g., yellow).

2. truecolor: created by converting the illumination wavelength for each band into the constituent RGB values as perceived by humans, then averaging the contribution to 

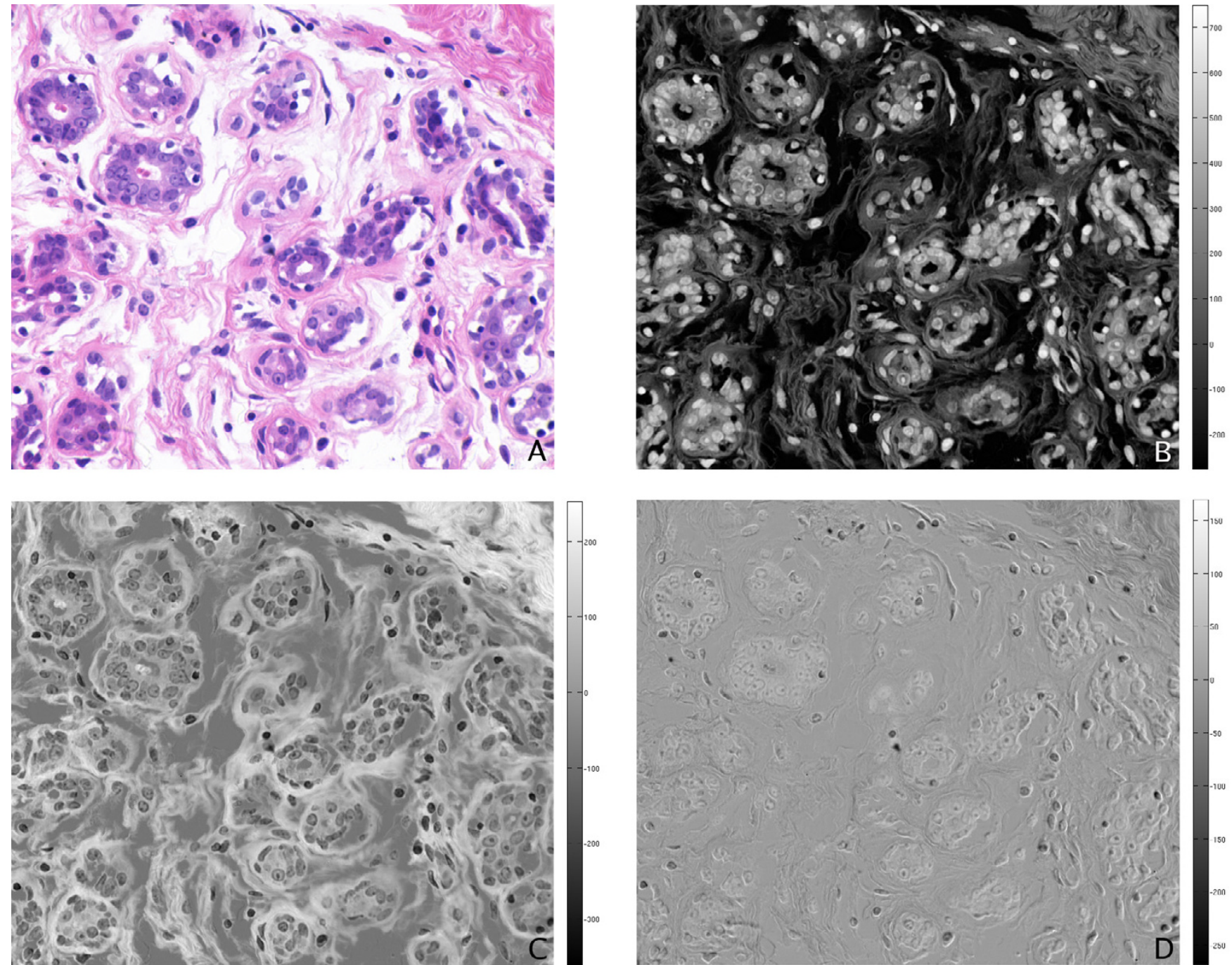

Figure 6

Projection of example image onto first three eigenvectors. (A) Projection on first eigenvector. (B) Projection on second eigenvector. (C) Projection on third eigenvector.

R, G, and B for each band. This method utilizes the MatlabCentral [20] function spectrumRGB.

3. ccd: a modification of truecolor imagery to better match the spectral response of common 3-CCD color cameras used in microscopy setups for biomedical research. This method also utilizes the spectrumRGB function.

It should be noted that the ccd and truecolor representations differ only in the red band. The RGB responses of the function spectrumRGB function are shown in Figure 8 and examples of each of these three types of RGB images are shown in Figure 9.

\section{Classifiers}

We describe here the six pixel-level classifiers used in this study. We choose these classifiers based on their established performance and use for multispectral data, sparsity of parameters to optimize, computational efficiency, and the use of (primarily) spectral information. The use of primarily spectral information is important in these analyses since the basic hypothesis in question deals with the spectral information content of our imagery. The exceptions to these characteristics are noted in the classifier descriptions to follow.

- Maximum Likelihood (ML) [21]: Maximizes the likelihood of a pixel belonging to a certain class. That is, a pixel is assigned the label of the class that it is most likely to be a member of. Likelihood is defined probabilistically, 
$420 \mathrm{~nm}$

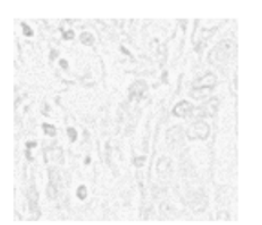

$480 \mathrm{~nm}$

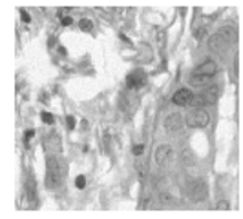

$540 \mathrm{~nm}$

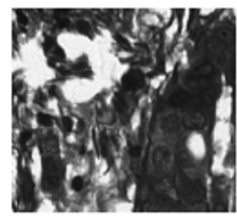

$600 \mathrm{~nm}$

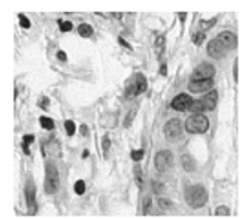

$660 \mathrm{~nm}$

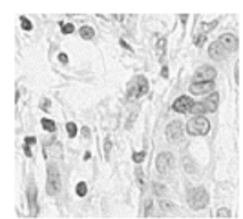

$430 \mathrm{~nm}$

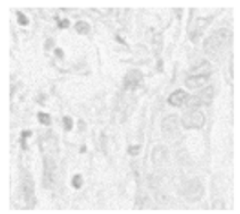

$490 \mathrm{~nm}$

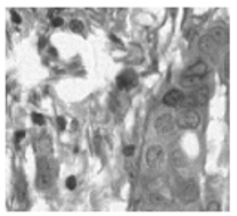

$550 \mathrm{~nm}$

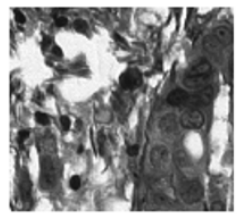

$610 \mathrm{~nm}$

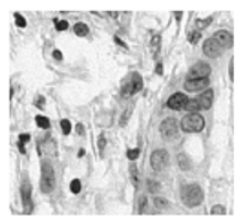

$670 \mathrm{~nm}$

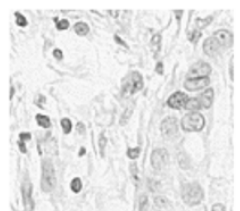

$440 \mathrm{~nm}$

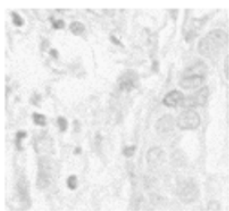

$500 \mathrm{~nm}$

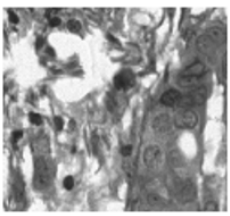

$560 \mathrm{~nm}$

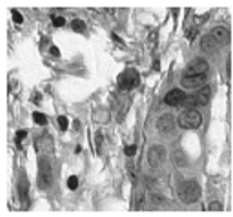

$620 \mathrm{~nm}$

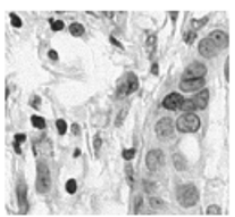

$680 \mathrm{~nm}$

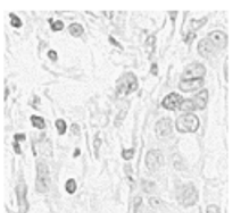

$450 \mathrm{~nm}$

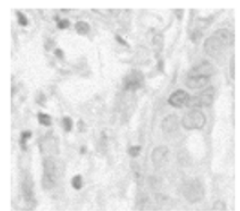

$510 \mathrm{~nm}$

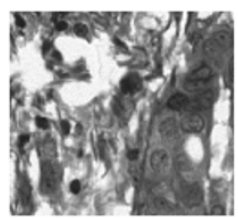

$570 \mathrm{~nm}$

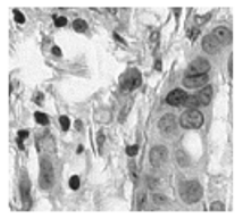

$630 \mathrm{~nm}$

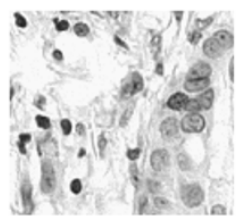

$690 \mathrm{~nm}$

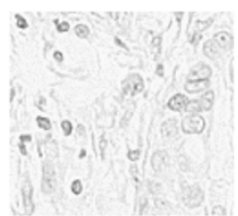

$460 \mathrm{~nm}$

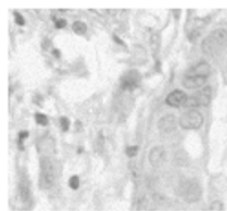

$520 \mathrm{~nm}$

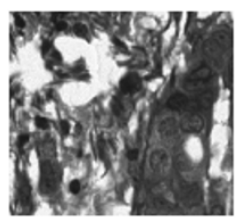

$580 \mathrm{~nm}$

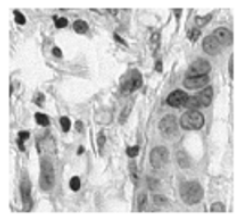

$640 \mathrm{~nm}$

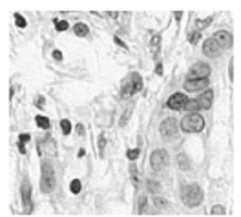

$700 \mathrm{~nm}$

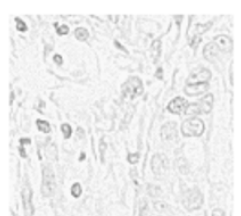

$470 \mathrm{~nm}$

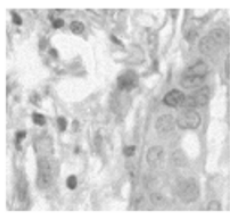

$530 \mathrm{~nm}$

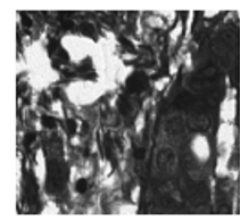

$590 \mathrm{~nm}$

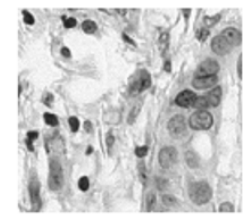

$650 \mathrm{~nm}$

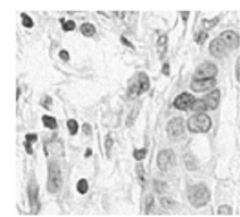

Figure 7

Example multispectral stack. Only a portion of the $768 \times 896$ image is shown.

using the estimated joint probability density or mass function. We assume a Gaussian density model, and estimate the mean and covariance matrix for each class. These assumptions result in a quadratic discrimination boundary.

- Minimum Euclidean Distance (MED): Minimizes the Euclidean distance between an observation and the class means.

- Spectral Angle Mapper (SAM): Minimizes the angle between an observation and the class means.

- Fisher Linear Discriminant Analysis (FLDA): Projects the multi-dimensional data to one dimension, maximizes a function representing the difference between the pro- jected class means, and normalizes by the within-class scatter along a direction perpendicular to the decision hyperplane [22]. This is also equivalent to a Maximum Likelihood formulation assuming equal covariance matrices for each class, resulting in a linear discrimination boundary.

- An Automated Feature Extraction (AFE) tool called GENIE: GENIE is based on evolutionary computation, and is designed to explore the entire feature space of multispectral data, and evolve a solution best fit for the classification task. More practically speaking, GENIE selects an initial set of algorithms consisting of randomly selected operators and randomly selected data planes as input. Throughout the evolution process, only appropriate algorithms with appropriate data input will survive. GENIE 


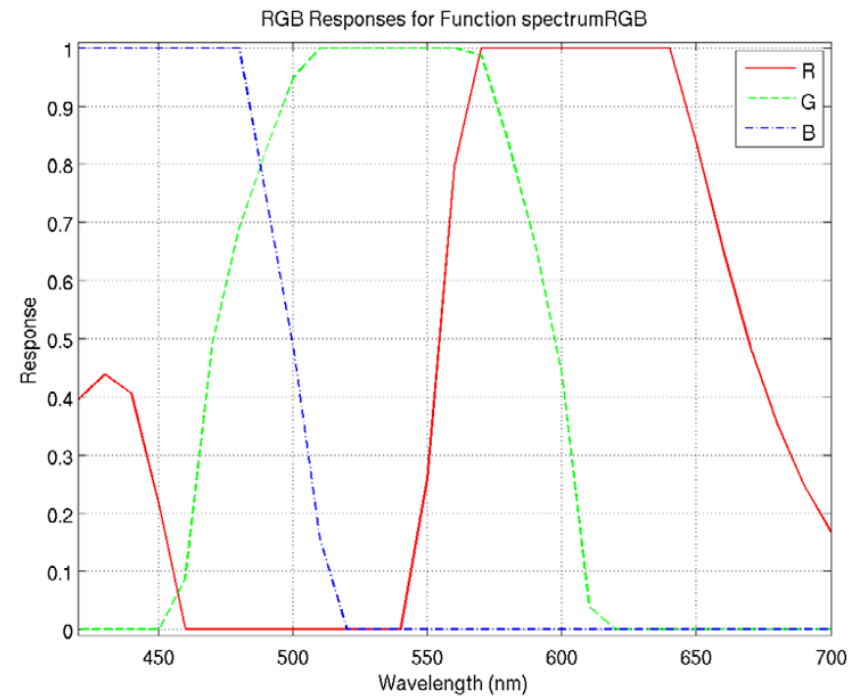

Figure 8

RGB responses for the function spectrumRGB. Note the second lobe of the red response in the smaller wavelengths. This is due to the perception of such wavelengths as violet, represented in RGB as a combination of red and blue. The ccd image representation removes the contribution of this second lobe.

has the ability to use information from both the spectral and spatial domain, which renders it unique among the six classifiers. For more information, see Reference [23].

- Support Vector Machine (SVM): Constructs a linear hyperplane that maximizes the margin between classes. In the case of nonlinear SVMs, the data is first mapped to a higher dimensional space where a linear hyperplane is computed to separate the classes, using a kernel function which defines the inner product operation in the higher dimensional space [24]. We have implemented an SVM using SVM ${ }^{\text {light }}$ [25], with a linear kernel (LSVM) using all training data as input, and a quadratic kernel (NLSVM) using a randomly selected $10 \%$ of our training data as input (to speed the training process to a reasonable time). For this classifier, the kernel parameters must be explicitly optimized for the training data; this is the only classifier used in this study which requires optimization of parameters.

Before discussing our performance metric and results, we would like to briefly discuss how these pixel-level nuclear classifications will be used. We are currently working towards a hierarchical image analysis system, where we will alternate classification and segmentation of the imagery in an interactive system eliciting user feedback. Current active research involves nuclear segmentation, i.e., the proper delineation of all nuclei contained in the image. As such, it is necessary to achieve an accurate classification of all nuclei pixels if we are to use shape and other appropriate metrics to their best advantage in the nuclear segmentation process.

Humans inherently incorporate higher-level information in their analysis of imagery; since we are considering the nuclear classification performance based on primarily spectral information, it is difficult, if not impossible, to specify the expected level of performance for a human expert. The issues of human performance in diagnosis, particularly the inter- and intra-observer variability (see $[26,27]$ and the references therein) will be an important consideration in our future work and is indeed a strong motivation for a computerized quantitative analysis.

\section{Performance metric}

We choose a general metric of classification performance that equally penalizes both types of classification errors: 1) true (nuclei) pixels incorrectly labeled as false (non-
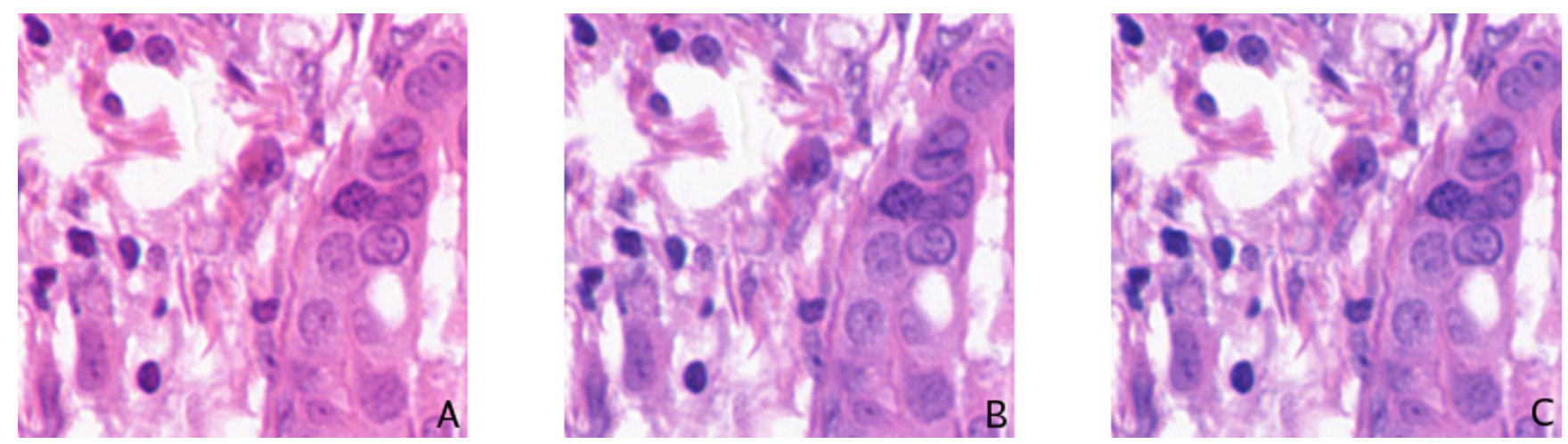

Figure 9

RGB representations of the example multispectral stack of Figure I. The same portion of the $768 \times 896$ image is shown here. (A) Bands allocated equally and averaged, "rgbequal" (B) Bands allocated with MatlabCentral function spectrum$R G B$, "truecolor." (C) Bands allocated to approximate spectral responses of common 3-CCD color cameras, "ccd." 
nuclei) and 2) false pixels incorrectly labeled as true. In particular, the performance metric is defined as

$$
P=0.5\left(R_{d}+\left(1-R_{f}\right)\right),
$$

where $R_{d}$ is the fraction of true pixels classified correctly (detection rate), $R_{f}$ is the fraction of false pixels classified incorrectly (false alarm rate), and the factor of 0.5 scales the metric to the range $[0,1]$. Note that a perfect segmentation will yield a performance score of $1(100 \%)$, while a score of $0.5(50 \%)$ can be obtained by a trivial solution of all pixels labeled as a single class (true or false). This metric is an equal tradeoff between detection rate and false alarm rate.

As a compromise between the necessity of comprehensive ground truth for proper quantification of classification accuracy, and the tedious and time-consuming aspect of human delineation of such ground truth, we have marked a $200 \times 200$ pixel window in each of our 58 histology images. This window is used to determine classification performance for each image.

\section{Abbreviations}

ML - Maximum Likelihood

MED - Minimum Euclidean Distance

SAM - Spectral Angle Mapper

FLDA - Fisher Linear Discriminant Analysis

AFE - Automated Feature Extraction

SVM - Support Vector Machine

LSVM - Linear Support Vector Machine

NLSVM - Non-Linear Support Vector Machine

PCA - Principal Components Analysis

ICA - Independent Components Analysis

\section{Competing interests}

The authors declare that they have no competing interests.

\section{Authors' contributions}

LEB performed analysis of the image data, including development of appropriate quantitative tests, necessary coding, and compilation and interpretation of the results, as well as drafting and revising the manuscript. ZB conducted the SVM classifications and provided technical expertise related to SVMs. NRH helped develop the test process, participated in the the interpretation of the results, and assisted with the preparation of the manuscript. BSM participated in the interpretation of results, suggestions for further analysis, and provided technical feedback for the final drafting of the manuscript. DLR provided pathology expertise, imagery, and technical feedback for the manuscript. All authors read and approved the final manuscript.

\section{Acknowledgements}

The authors would like to gratefully acknowledge James Theiler, Steven Brumby, Reid Porter, and Jiyun Byun for their excellent ideas and feedback about our test design and results, as well as Carola Zalles for her pathology tutelage.

LEB would like to acknowledge her funding support from NSF IGERT Grant DGE-022 I7/ 3 for her first two years of Ph.D. research, and Los Alamos National Laboratory for subsequent funding.

This article has been published as part of BMC Cell Biology Volume 8 Supplement I, 2007: 2006 International Workshop on Multiscale Biological Imaging, Data Mining and Informatics. The full contents of the supplement are available online at http://www.biomedcentral.com/|47|-2/2|/8?issue=SI

\section{References}

I. Levenson RM, Wachman ES, Niu W, Farkas DL: Spectral imaging in biomedicine: A selective overview. Proc SPIE 1998, 3438:300-312.

2. Levenson RM: Spectral imaging and pathology: seeing more. Laboratory Medicine 2004, 35(4):244-25I.

3. Jaganath R, Angeletti C, Levenson R, Rimm DL: Diagnostic classification of urothelial cells in urine cytology specimens using exclusively spectral information. Cancer Cytopathology 2004, 102(3): |86-19|.

4. Angeletti C, Harvey NR, Khomitch V, Fischer AH, Levenson RM, Rimm DL: Detection of malignancy in cytology specimens using spectral-spatial analysis. Lab Invest 2005, 85: I555-I 564.

5. Levenson R, Cronin PJ, Pankratov KK: Spectral imaging for brightfield microscopy. Proc SPIE 2003, 4959:27-33.

6. Roula MA, Bouridane A, Kurugollu F, Amira A: A quadratic classifier based on multispectral texture features for prostate cancer diagnosis. Proc Int Symp on Signal Processing and lts Applications 2003, 2:37-40.

7. Gentry SM, Levenson R: Biomedical applications of the information-efficient spectral imaging sensor (ISIS). Proc SPIE 1999, 3603:129-142.

8. Anderson NH, Hamilton PW, Bartels PH, Thompson D, Montironi R, Sloan JM: Computerized scene segmentation for the discrimination of architectural features in ductal proliferative lesions of the breast. J Pathol 1997, 181:374-380.

9. Brewer MA, Ranger-Moore J, Greene MH, Alberts DS, Liu Y, Bartels HG, Baruch AC, Bartels PH: Preneoplastic changes in ovarian tissues. Anal Quant Cytol Histol 2004, 26(4):207-216.

10. Neher A, Öfner G, Appenroth E, Gschwendtner A: High-resolution image cytometry on smears of normal oral mucosa: $A$ possible approach for the early detection of laryngopharyngeal cancers. Head Neck 2004, 26:694-700.

II. Zahniser DJ, Isenstein LM, Hutchinson ML: Computerized contextual analysis in diagnosis. Cancer Lett 1994, 77:139-144.

12. Mairinger T, Mikuz G, Gschwendter A: Nuclear chromatin texture analysis of nonmalignant tissue can detect adjacent prostatic adenocarcinoma. Prostate 1999, $41: 12-19$.

13. van de Wouwer G, Weyn B, Scheunders P, Jacob W, van Marck E, van Dyck D: Wavelets as chromatin texture descriptors for the automated identification of neoplastic nuclei. J Microsc 2000, 197:25-35.

14. Weyn B, van de Wouwer G, Koprowski M, van Daele A, Dhaene K, Scheunders P, Jacob W, van Marck E: Value of morphometry, texture analysis, densitometry, and histometry in the differen- 
tial diagnosis and prognosis of malignant mesothelioma. J Pathol 1999, 189:58I-589.

15. Weyn B, van de Wouwer G, Kumar-Singh S, van Daele A, Scheunders $P$, van Marck E, Jacob W: Computer-assisted differential diagnosis of malignant mesothelioma based on syntactic structure analysis. Cytometry 1999, 35:23-29.

16. Weyn B, van de Wouwer $G$, van Daele A, Scheunders P, van Dyck D, van Marck E, Jacob W: Automated breast tumor diagnosis and grading based on wavelet chromatin texture description. Cytometry 1998, 33:32-40.

17. Boucheron LE, Harvey NR, Manjunath BS: Utility of multispectral imaging for analysis of routine clinical histopathology imagery. 2006. Presented at the 2006 Workshop on Multiscale Biological Imaging, Data Mining, \& Informatics, Santa Barbara, CA

18. Bartlett MS, Lades HM, Sejnowski T]: Independent component representations for face recognition. Proc SPIE 1998, 3299:528-539.

19. Yale University School of Medicine Tissue Microarray Facility [http://tissuearray.org/facility/inventory/list.php]

20. MATLAB Central, An open exchange for the MATLAB and Simulink user community [http://www.mathworks.com/matlab central/fileexchange/]

21. Stark H, Woods JW: Probability and Random Processes with Applications to Signal Processing 3rd edition. Prentice Hall; 2002.

22. Bishop CM: Neural Networks for Pattern Recognition Oxford University Press; 1995.

23. Harvey NR, Theiler J, Brumby SP, Perkins S, Szymanski JJ, Bloch JJ, Porter RB, Galassi M, Young AC: Comparison of GENIE and conventional supervised classifiers for multispectral image feature extraction. IEEE T Geosci Remote 2002, 40:393-404.

24. Theodoridis S, Koutroumbas K: Pattern Recognition 2nd edition. Elselvier Academic Press; 2003.

25. SVM-Light Support Vector Machine [http://svm light.joachims.org/]

26. Latson L, Sebek B, Powell KA: Automated cell nuclear segmentation in color images of hematoxylin and eosin-stained breast biopsy. Anat Quant Cytol Histol 2003, 25:32I-33I.

27. Schnorrenberg F, Pattichis CS, Kyriacou KC, Schizas CN: Computer-aided detection of breast cancer nuclei. IEEE Trans Inf Technol Biomed 1997, I: 128-140.

Publish with Bio Med Central and every scientist can read your work free of charge

"BioMed Central will be the most significant development for disseminating the results of biomedical research in our lifetime. "

Sir Paul Nurse, Cancer Research UK

Your research papers will be:

- available free of charge to the entire biomedical community

- peer reviewed and published immediately upon acceptance

- cited in PubMed and archived on PubMed Central

- yours - you keep the copyright 